\title{
Comparison of immediate vs. deferred cytoreductive nephrectomy in patients with synchronous metastatic renal cell carcinoma
}

\author{
Brian W. Cross, Michael S. Cookson \\ Department of Urology, University of Oklahoma, Oklahoma City, OK 73104, USA.
}

Correspondence to: Dr. Michael S. Cookson, Department of Urology, University of Oklahoma, 920 SL Young Blvd, WP 2140, Oklahoma City, OK 73104, USA. E-mail: michael-cookson@ouhsc.edu

How to cite this article: Cross BW, Cookson MS. Comparison of immediate vs. deferred cytoreductive nephrectomy in patients with synchronous metastatic renal cell carcinoma. J Cancer Metastasis Treat 2021;7:55. https://dx.doi.org/10.20517/23944722.2021 .99

Received: 20 Apr 2021 First Decision: 19 May 2021 Revised: 7 Jun 2021 Accepted: 29 Jun 2021 Published: 5 Sep 2021

Academic Editor: Hendrik Van Poppel Copy Editor: Yue-Yue Zhang Production Editor: Yue-Yue Zhang

\begin{abstract}
Cytoreductive nephrectomy has been a mainstay in treating patients with synchronous metastatic renal cell carcinoma (mRCC) for over two decades. It was supported in part by level 1 evidence that showed improved survival for patients undergoing radical nephrectomy before initiation of systemic therapy dating back almost 20 years. Since that time, the landscape of systemic therapy for mRCC has shifted mainly from IL-2 based therapy to tyrosine kinase inhibitors (TKIs) targeting the vascular endothelial growth factor pathway, and now to immunotherapy with PD-L1 inhibitors. Given the significant advancements in systemic therapy for patients with mRCC, the role of cytoreductive nephrectomy and sequencing of treatment has been questioned. Recent randomized studies appear to disprove the theory that upfront cytoreduction improves overall survival, particularly in the TKI era, and thus treating physicians are faced with conflicting data to guide treatment decisions. The role of cytoreductive nephrectomy is in evolution, and so is the timing of surgery in selected patients. Familiarity with available evidence coupled with patient selection and targeted therapy should help to inform decision-making. Currently, an initial course of systemic therapy followed by consideration of nephrectomy in those with a favorable response may be the most prudent algorithm outside the context of a clinical trial.
\end{abstract}

Keywords: Metastatic kidney cancer, immediate vs. delayed cytoreductive nephrectomy, survival 


\section{INTRODUCTION}

Approximately 400,000 new cases of renal cell carcinoma (RCC) are diagnosed worldwide each year, with a resultant 175,000 deaths $^{[1]}$. Of these, approximately $15 \%-20 \%$ of patients will present with synchronous metastatic disease, that is, patients diagnosed with a primary kidney tumor and a corresponding distant metastatic site ${ }^{[2]}$. Until recently, patients diagnosed with synchronous metastatic renal cell carcinoma (mRCC) who were fit for surgery were offered upfront cytoreductive nephrectomy as standard-of-care treatment. This was based on two widely cited randomized controlled trials comparing cytoreductive nephrectomy plus interferon alfa to interferon alfa alone ${ }^{[3,4]}$. Both of these trials were published in 2001 and demonstrated survival-benefit for patients undergoing upfront cytoreductive nephrectomy in the setting of synchronous metastatic disease. Flanigan et al..$^{[3]}$ showed a 3-month survival advantage among patients undergoing nephrectomy followed by interferon therapy compared to interferon alone, while Mickisch et al. ${ }^{[4]}$ demonstrated a delayed time to progression and improved overall survival as well. These studies served as the backbone of treatment algorithms for patients with synchronous mRCC for nearly two decades.

Over time, however, the treatment landscape of systemic therapy for patients with mRCC changed considerably, particularly with the introduction of therapies targeting RCC molecular tumorigenesis pathways in $2005^{[5]}$. With this significant shift away from interferon and IL-2-based systemic therapies toward drugs targeting RCC carcinogenesis, particularly the vascular endothelial growth factor (VEGF) receptor tyrosine kinase inhibitors (TKIs) as well as the anti-VEGF monoclonal antibody bevacizumab, the role of upfront cytoreductive nephrectomy was once again questioned. Indeed, there was a lack of level 1 evidence comparing immediate cytoreductive nephrectomy to targeted therapies alone. Further, given that most patients presenting with synchronous metastatic disease have locally advanced primary tumors, recovery from surgery often takes several weeks, if not more. This postoperative recovery potentially delays the initiation of systemic therapy, with the potential to negatively impact the outcome. It was the impetus for the newly published randomized trials comparing cytoreductive nephrectomy to TKI monotherapy. This review will address the controversies regarding cytoreductive nephrectomy and analyze the best available data to guide treatment decisions.

\section{EARLY CYTOREDUCTIVE NEPHRECTOMY TRIALS}

Any review addressing the role of cytoreductive nephrectomy in patients with synchronous mRCC would be remiss without mentioning the first trials randomizing patients to cytoreductive nephrectomy $v s$. systemic monotherapy. While these trials are mostly considered historical at this point, they laid the groundwork for cytoreductive nephrectomy in the treatment algorithm of patients with mRCC for nearly two decades.

In 2001, Mickisch et al. ${ }^{[4]}$ published the first randomized trial of cytoreductive nephrectomy in The Lancet (EORTC 30947), randomizing 85 patients with synchronous mRCC to radical nephrectomy plus interferon alfa immunotherapy $v s$. interferon alone. The primary endpoints were time to progression and overall survival. The study showed a modest but statistically significant improvement in time to progression for the study group compared to the control group (5 months $v s .3$ months), as well as an improved overall survival for patients undergoing nephrectomy (17 months $v s .7$ months).

Later in 2001, Flanigan et al. ${ }^{[3]}$ published the concurrently performed randomized SWOG trial of nephrectomy followed by interferon-alfa compared with interferon-alfa alone in The New England Journal of Medicine. SWOG 8949 randomized 246 patients with mRCC to either radical nephrectomy followed by therapy with interferon-alfa- $2 \mathrm{~b}$ or to receive interferon-alfa- $2 \mathrm{~b}$ therapy alone. The study's primary endpoint 
was overall survival with a secondary endpoint of tumor response to treatment. Similar to the EORTC counterpart, the SWOG study showed a statistically significant improvement in overall survival in patients undergoing cytoreductive nephrectomy, though the observed improvement in survival in the SWOG study was less, 11 months $v s .8$ months.

Three years later, in 2004, a combined analysis of both of the preceding randomized cytoreductive nephrectomy trials was published by Flanigan et al. ${ }^{[6]}$ in The Journal of Urology. This combined effort by SWOG and EORTC analyzed the combined 331 patients randomized in both studies to upfront cytoreductive nephrectomy followed by interferon alfa $v$ s. interferon alfa monotherapy. The primary endpoints of overall survival and secondary endpoints of response to therapy were evaluated. The combined analysis showed an overall median survival of 13.6 months for patients undergoing nephrectomy followed by interferon alfa vs. 7.8 months for interferon alone. It represented a $31 \%$ decrease in the risk of death in the surgery arm. The Kaplan-Meier estimate of 1-year survival was $51.9 \%$ in the study group (nephrectomy plus interferon) vs. $37.1 \%$ in the control group (interferon alone). Figure 1 shows the unadjusted survival curves for the 2 treatment regimens.

While these trials are mainly considered from a historical perspective in the current treatment landscape of patients presenting with upfront mRCC, their importance cannot be overstated, as they cemented cytoreductive nephrectomy as a part of the treatment algorithm for patients with synchronous mRCC for nearly 20 years. They were the only randomized level 1 data on which treating physicians had to rely on making treatment decisions.

\section{CHANGING LANDSCAPE OF SYSTEMIC THERAPY}

In the mid-2000s, an improved understanding of the molecular pathways of RCC carcinogenesis led to the FDA approval of several new targeted therapies, including vascular endothelial growth factor neutralizing antibodies, tyrosine kinase inhibitors, and mammalian target of rapamycin inhibitors ${ }^{[7,8]}$. It led some physicians to question the continued need for upfront cytoreductive nephrectomy prior to initiation of systemic therapy, particularly in light of these dramatic improvements in the systemic treatment of these patients.

In response to this shift away from interferon and IL-2-based immunotherapy toward systemic therapy targeting the biology of RCC tumorigenesis, numerous multi-institutional retrospective trials were undertaken to evaluate the benefit of cytoreductive nephrectomy again. While there are far too many retrospective studies to cover concisely in this review, a few warrant discussions.

In 2011, Choueiri et al. ${ }^{[9]}$ published a multi-institutional retrospective trial from 7 different oncology treatment centers across the United States and Canada, analyzing overall survival in 314 patients with metastatic RCC receiving VEGF-target therapy with either sunitinib, sorafenib, or bevacizumab. Of the 314 patients included in the analysis, 201 underwent cytoreductive nephrectomy, and 113 did not. The median time from surgery to the start of targeted therapy was 5 months. Not surprisingly, patients who underwent cytoreductive nephrectomy were overall younger with better performance status. On univariable analysis, cytoreductive nephrectomy was associated with a median overall survival of 19.8 months compared to 9.4 months for patients who did not undergo surgery. On multivariable analysis, patients with poor-risk criteria based on Memorial Sloan Kettering (MSKCC) and International Metastatic RCC Database Consortium (IMDC) criteria ${ }^{[10,11]}$ and those with poor performance status (Karnofsky performance status $<80$ ) seemed to have marginal survival benefit from cytoreductive nephrectomy. 


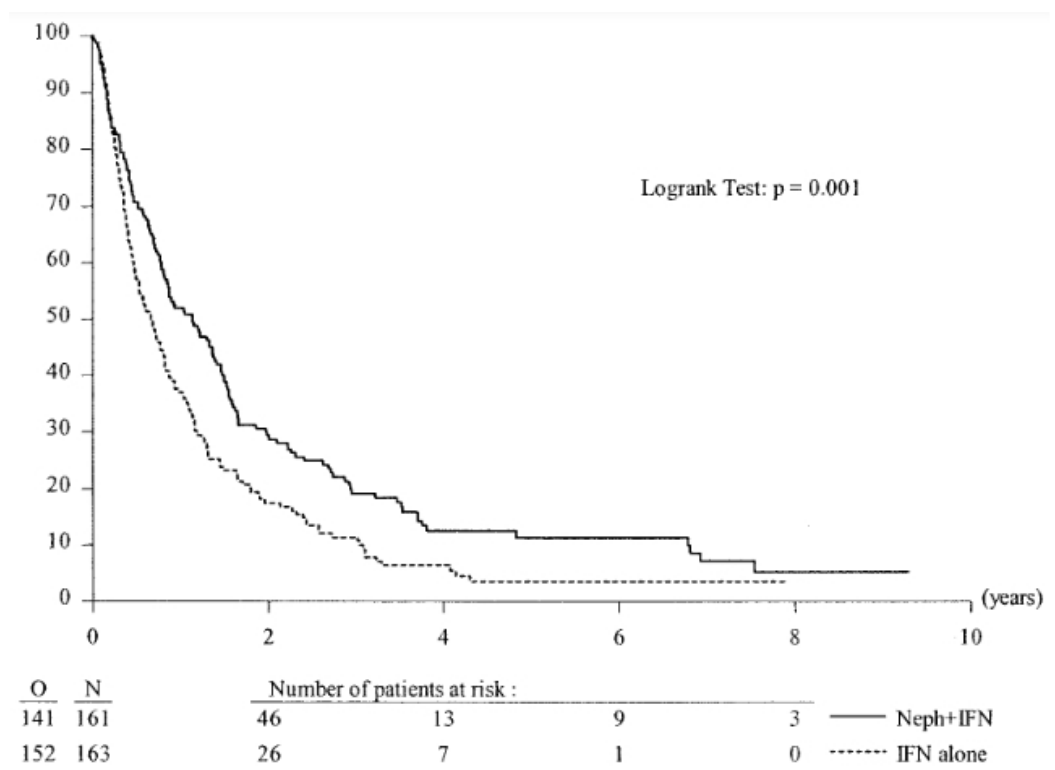

Figure 1. Duration of survival in combined SWOG and EORTC trails. Unadjusted survival curves for nephrectomy followed by interferon vs. interferon alone for patients with synchronous mRCC. O: Observation; N: nephrectomy. From Flanigan et al. ${ }^{[6]}$, J Urol March 2004.

Three years later, in 2014, Heng et al.$^{[12]}$ published their results from the IMDC. Also retrospective in nature, this study analyzed the overall survival benefit of cytoreductive nephrectomy in patients treated with targeted therapies. Retrospective data were analyzed from patients at 20 international cancer centers from Canada, the United States, Belgium, South Korea, Japan, Denmark, Greece, and Singapore. Included patients diagnosed with mRCC and treatment with a VEGF or mTOR targeted therapy (sunitinib, sorafenib, axitinib, bevacizumab, temsirolimus, pazopanib, or everolimus). In total, 1658 patients met inclusion criteria, including 982 who underwent nephrectomy vs. 676 who did not. The median overall survival for patients undergoing cytoreductive nephrectomy was 20.6 months $v s .9 .6$ months for those who did not have surgery. Progression-free survival was also improved in the nephrectomy group, 7.6 months vs. 4.5 months. As discussed in the prior study, when stratified by IMDC risk criteria, those poor-risk patients with multiple adverse prognostic factors $(>3)$ did not seem to derive benefit from cytoreductive nephrectomy.

More recently, in 2016, Hanna et al ${ }^{[13]}$ published a review of the National Cancer Database analyzing 15,390 patients with metastatic RCC treated with targeted therapy, 5374 (35\%) of whom underwent cytoreductive nephrectomy. Patients undergoing cytoreductive nephrectomy were younger $(<50$ years) with minimal comorbidities (Charlson comorbidity index 0). Meantime to death was 32.5 months for patients undergoing nephrectomy vs. 14.9 months for those not undergoing surgery.

These are but three of the numerous retrospective studies undertaken after the advent of improved systemic therapies aimed at defining the role of cytoreductive nephrectomy in the targeted therapy era. While cytoreductive nephrectomy had been cemented in the treatment algorithm of patients with mRCC since the immunotherapy era (1992-2004) based on level 1 data, all treating physicians had to rely upon these retrospective data to decide if nephrectomy was still beneficial. While these studies were well-done and provided valuable information, retrospective case-control studies suffer from nearly insurmountable selection bias. Patients undergoing cytoreductive nephrectomy included in retrospective studies were likely younger, healthier, and with better risk profile, which may skew the results to favor cytoreductive nephrectomy. 


\section{CONTEMPORARY CYTOREDUCTIVE NEPHRECTOMY TRIALS}

In response to the lack of high-quality data to guide treatment decisions regarding cytoreductive nephrectomy in patients receiving VEGF and mTOR targeted therapy, prospective clinical trials evaluating cytoreductive nephrectomy were sorely needed. CARMENA and SURTIME were two randomized controlled trials initiated in 2010 to investigate the necessity of cytoreductive nephrectomy in the era of VEGF tyrosine kinase inhibitors. Each of these warrants in-depth discussion.

\section{SURTIME}

The first of these two trials, the SURTIME (Immediate Surgery or Surgery After Sunitinib Malate in Treating Patients With Metastatic Kidney Cancer) trial, was jointly conducted by the EORTC GenitoUrinary Cancer Group, the National Cancer Research Institute Renal Clinical Studies Group/Wales Cancer Trial Unit (United Kingdom), and the Canadian Uro-Oncology Group beginning in $2010^{[14]}$. The open-label multicenter trial randomized patients 1:1 between immediate cytoreductive nephrectomy followed by sunitinib therapy $v$ s. treatment with 3 cycles of sunitinib followed by restaging at 16 weeks followed by cytoreductive nephrectomy. In the case of progressive disease, proceeded decision with nephrectomy was left at the discretion of each investigator.

The study had a goal accrual of 450 patients but fell well short of that goal, randomizing 99 patients over 6 years at 19 institutions throughout Europe and Canada. The primary endpoint was progression-free survival (PFS), but this had to be altered to PFS at 28 weeks due to poor accrual. Fifty patients were randomized to immediate nephrectomy vs. 49 to deferred nephrectomy. The median follow-up was 3.3 years. Patients were predominantly MSKCC intermediate-risk (88\%). The 28 -week progression-free rate was $42 \%$ in the immediate surgery group vs. $43 \%$ in the deferred surgery arm [Figure 2].

Although the study did not meet its primary endpoint, the investigators did note a difference in overall survival, which served as a secondary endpoint. Patients in the deferred nephrectomy group had an overall survival of 32 months, compared with 15 months for those in the immediate nephrectomy group. While the study is likely underpowered to prove that delayed nephrectomy is beneficial, we can surmise that with the TKIs, there will be patients who progress through systemic therapy and are clearly not going to do well with upfront surgery. A course of upfront systemic therapy may identify these patients, and they can be spared the morbidity and recovery of cytoreductive nephrectomy.

\section{CARMENA}

Parallel to SURTIME, the CARMENA trial (Cancer du Rein Metastatique Nephrectomie et Antiangiogé niques), a phase 3 non-inferiority trial, was conducted between 2009 and 2017 at 79 centers, led by European investigators ${ }^{[15]}$. In this trial, 450 patients with confirmed metastatic clear-cell RCC and ECOG performance status of 0-1 were randomized 1:1 to undergo nephrectomy and then receive sunitinib or to receive sunitinib alone. All patients had MSKCC intermediate-risk (one or two prognostic factors) or poorrisk disease (three or more prognostic factors). The primary endpoint of the trial was overall survival.

After about 50 months of follow-up, an interim analysis of the intention-to-treat population showed median OS times of 18 months in the sunitinib-only group $v s .14$ months in the cytoreductive nephrectomy group. Based on this interim analysis and the trial's slow accrual, the decision was made to close the trial early [Figure 3].

One of the main criticisms of the study was that many of the patients in the CARMENA trial were not treated as planned. In the cytoreductive nephrectomy arm, 7\% of patients did not receive nephrectomy, and 
A Progression-free survival

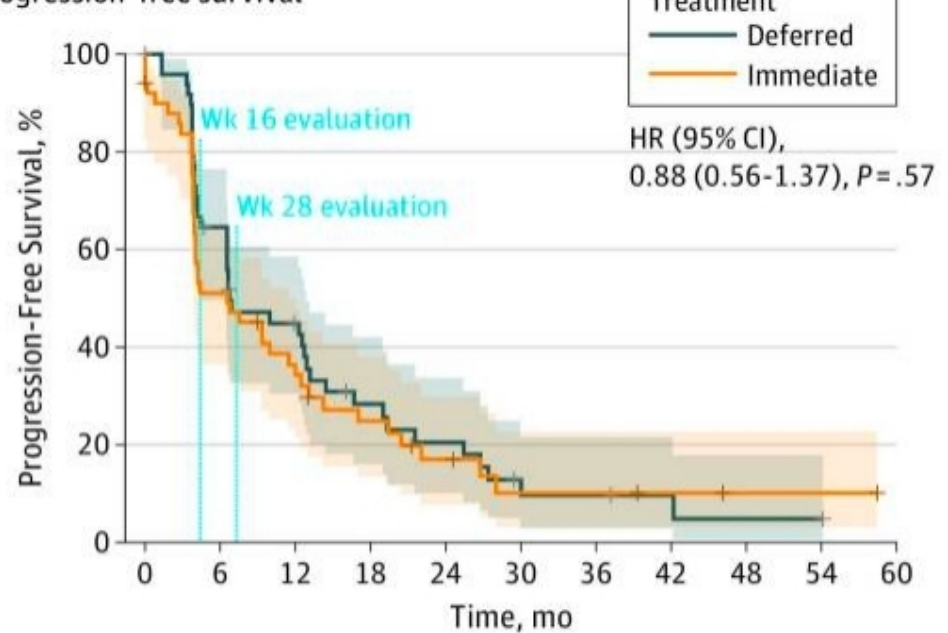

No. at risk

Treatment

$\begin{array}{llllllllllll}\text { Deferred } & 49 & 30 & 19 & 11 & 8 & 3 & 3 & 2 & 1 & 1 & 0\end{array}$

Immediate $\begin{array}{lllllllllll}50 & 25 & 16 & 10 & 6 & 3 & 3 & 2 & 1 & 1 & 0\end{array}$

Figure 2. Progression-free survival of immediate vs. deferred cytoreductive nephrectomy in the SURTIME trial.

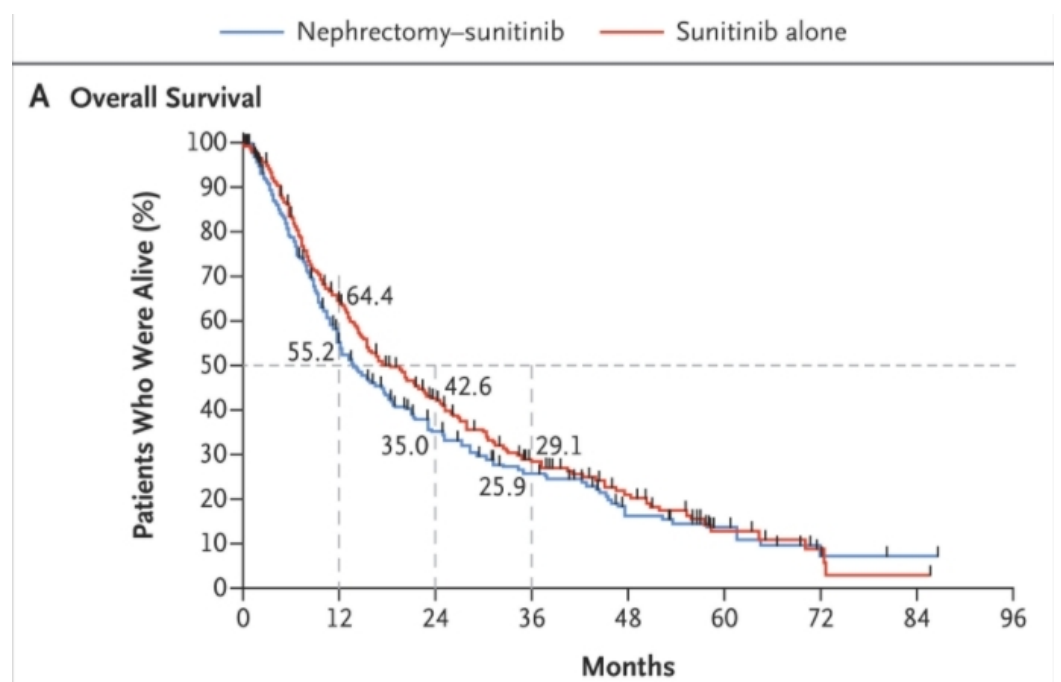

\section{No. at Risk}

$\begin{array}{ccccccccc}\begin{array}{c}\text { Nephrectomy- 226 } \\ \text { sunitinib }\end{array} & 110 & 61 & 40 & 19 & 11 & 4 & 1 & 0 \\ \begin{array}{c}\text { Sunitinib alone 224 } 22 \\ \text { unition }\end{array} & 128 & 76 & 44 & 26 & 8 & 3 & 1 & 0\end{array}$

Figure 3. Kaplan-Meier estimates of overall survival comparing nephrectomy plus sunitinib vs. sunitinib alone in the CARMENA trial.

an additional $18 \%$ never received systemic therapy. In the sunitinib-only arm, $5 \%$ of patients didn't receive the planned systemic therapy, and an additional 17\% subsequently underwent nephrectomy. A per-protocol analysis including only those patients who were treated as assigned showed a median OS of 20.5 months for the sunitinib-only group vs. 18.3 months in the cytoreductive nephrectomy group. Based on this analysis, making definitive conclusions from the study is more difficult. 
Further criticism of the study included the fact that $>40 \%$ of the patients were MSKCC poor-risk and probably would not be offered cytoreductive nephrectomy at most institutions, likely skewing the results away from nephrectomy. It could explain why the overall survival in CARMENA was shorter than in other studies of metastatic kidney cancer. Additionally, CARMENA patients had a significant metastatic disease burden, comprising at least $40 \%$ of the overall tumor burden for most patients. If one of the primary goals of cytoreductive nephrectomy is to debulk the tumor burden significantly, this would not have been possible for these patients. Patients with low metastatic disease burden were excluded from CARMENA at the investigator's discretion, probably because most agree that those with low metastatic disease burden would benefit from upfront nephrectomy. It also likely skewed the trial away from showing a benefit to cytoreductive nephrectomy.

Lastly, CARMENA and SURTIME suffer from the same fate that ultimately led many physicians to question the continued validity of the early cytoreductive nephrectomy trials. That is, sunitinib is no longer standard first-line therapy for patients who present with synchronous metastatic kidney cancer. With the results of recent randomized trials, first-line therapy for patients with metastatic kidney cancer now consists of combination immunotherapy with either ipilimumab plus nivolumab (CheckMate 214) or axitinib plus pembrolizumab (KEYNOTE-426) $)^{[16,17]}$.

\section{PROBE trial}

The recently activated Phase III trial of nivolumab plus ipilimumab with or without cytoreductive nephrectomy for metastatic renal cell carcinoma (PROBE trial, SWOG 1931) will attempt to answer the question regarding the benefit of cytoreductive nephrectomy in the immunotherapy era. As with the previous trials, the primary outcome will be overall survival with an estimated enrollment of 364 patients and a completion date of July 2033. Patients will receive PD-L1 inhibition combination therapy for 12-18 weeks, followed by updated imaging to evaluate their response. Patients with a partial response or stable disease will then be randomized 1:1 to cytoreductive nephrectomy vs. continued immunotherapy (https://clinicaltrials.gov/ct2/show/NCT04510597).

\section{SUMMARY}

In summary, the role of cytoreductive nephrectomy in the treatment algorithm of patients presenting with synchronous metastatic RCC has evolved considerably since the original randomized trials were published in 2001. While those original trials showed improved overall survival of up to 10 months, more recent trials have called into question the blanket utilization of upfront nephrectomy in these patients. Others have advocated for an initial course of systemic therapy followed by nephrectomy in those with a favorable response. In particular, the CARMENA trial failed to show a benefit to cytoreductive nephrectomy when combined with systemic therapy.

Certainly, those patients who progress through an upfront course of immunotherapy are unlikely to benefit from cytoreductive nephrectomy, and a trial of systemic therapy may save them the morbidity of the surgery. The deferred nephrectomy strategy is partly supported by data from the SURTIME trial, which showed an improved overall survival as a secondary endpoint.

The current risk stratification models are suboptimal to assess patients who present with de novo metastatic disease. Since even one prognostic point puts patients in an intermediate-risk category in either the MSKCC or IMDC criteria, and both take the time between diagnosis and systemic therapy into account, those who present with synchronous metastatic disease cannot be classified as good-risk. The current prognostic models were designed for patients who have already undergone nephrectomy and subsequently develop 
metachronous metastatic disease. It underscores the importance of updated patient selection criteria when considering patients for cytoreductive nephrectomy.

Appropriate patient selection criteria for cytoreductive nephrectomy cannot be overstated. Certainly, those with poor performance status who require prolonged recovery from surgery before beginning systemic therapy are unlikely to benefit. Further, those with a high metastatic disease burden outside the kidney and who cannot be significantly debulked are also likely best served with upfront systemic therapy. Alternatively, those with symptoms from the primary tumor (pain or hematuria) are likely to benefit from nephrectomy, if just for palliative reasons.

The question remains for those with good performance status and limited metastatic disease burden. These are the patients who are potentially most likely to benefit from removing their primary tumor, but highquality evidence is needed in the current immunotherapy era. While the upcoming SWOG trial will hopefully answer some of these questions, the discrepancy between the rapid advances in systemic therapy and the time necessary to complete and analyze randomized surgical trials (i.e., a decade or more), cytoreductive nephrectomy may remain an operation without high-quality, up-to-date data to guide clinical decision making.

\section{DECLARATIONS}

\section{Authors' contributions}

Responsible for the paper, concept, design, literature search, manuscript preparation and editing: Cross BW, Cookson MS

\section{Availability of data and materials}

Not applicable.

\section{Financial support and sponsorship}

None.

\section{Conflicts of interest}

Both authors declared that there are no conflicts of interest.

\section{Ethical approval and consent to participate}

Not applicable.

\section{Consent for publication}

Not applicable.

\section{Copyright}

(c) The Author(s) 2021.

\section{REFERENCES}

1. Bray F, Ferlay J, Soerjomataram I, Siegel RL, Torre LA, Jemal A. Global cancer statistics 2018: GLOBOCAN estimates of incidence and mortality worldwide for 36 cancers in 185 countries. CA Cancer J Clin 2018;68:394-424. DOI PubMed

2. Siegel RL, Miller KD, Jemal A. Cancer statistics, 2018. CA Cancer J Clin 2018;68:7-30. DOI PubMed

3. Flanigan RC, Salmon SE, Blumenstein BA, et al. Nephrectomy followed by interferon alfa-2b compared with interferon alfa-2b alone for metastatic renal-cell cancer. N Engl J Med 2001;345:1655-9. DOI PubMed

4. Mickisch G, Garin A, van Poppel H, de Prijck L, Sylvester R. Radical nephrectomy plus interferon-alfa-based immunotherapy compared with interferon alfa alone in metastatic renal-cell carcinoma: a randomised trial. Lancet 2001;358:966-70. DOI PubMed

5. Choueiri TK, Motzer RJ. Systemic Therapy for Metastatic Renal-Cell Carcinoma. N Engl J Med 2017;376:354-66. DOI PubMed

6. Flanigan RC, Mickisch G, Sylvester R, Tangen C, Van Poppel H, Crawford ED. Cytoreductive nephrectomy in patients with 
metastatic renal cancer: a combined analysis. J Urol 2004;171:1071-6. DOI PubMed

7. Choueiri TK, Bukowski RM, Rini BI. The current role of angiogenesis inhibitors in the treatment of renal cell carcinoma. Semin Oncol 2006;33:596-606. DOI PubMed

8. Heng DY, Kollmannsberger C. State-of-the-art treatment of metastatic renal cell carcinoma. Curr Oncol 2009;16 Suppl 1:S16-23. DOI PubMed PMC

9. Choueiri TK, Xie W, Kollmannsberger C, et al. The impact of cytoreductive nephrectomy on survival of patients with metastatic renal cell carcinoma receiving vascular endothelial growth factor targeted therapy. J Urol 2011;185:60-6. DOI PubMed

10. Heng DY, Xie W, Regan MM, et al. External validation and comparison with other models of the International Metastatic Renal-Cell Carcinoma Database Consortium prognostic model: a population-based study. Lancet Oncol 2013;14:141-8. DOI PubMed PMC

11. Motzer RJ, Mazumdar M, Bacik J, Berg W, Amsterdam A, Ferrara J. Survival and prognostic stratification of 670 patients with advanced renal cell carcinoma. J Clin Oncol 1999;17:2530-40. DOI PubMed

12. Heng DY, Wells JC, Rini BI, et al. Cytoreductive nephrectomy in patients with synchronous metastases from renal cell carcinoma: results from the International Metastatic Renal Cell Carcinoma Database Consortium. Eur Urol 2014;66:704-10. DOI PubMed

13. Hanna N, Sun M, Meyer CP, et al. Survival analyses of patients with metastatic renal cancer treated with targeted therapy with or without cytoreductive nephrectomy: a national cancer data base study. J Clin Oncol 2016;34:3267-75. DOI PubMed PMC

14. Bex A, Mulders P, Jewett M, et al. Comparison of immediate vs deferred cytoreductive nephrectomy in patients with synchronous metastatic renal cell carcinoma receiving sunitinib: the SURTIME randomized clinical trial. JAMA Oncol 2019;5:164-70. DOI PubMed PMC

15. Méjean A, Ravaud A, Thezenas S, et al. Sunitinib alone or after nephrectomy in metastatic renal-cell carcinoma. $N$ Engl J Med 2018;379:417-27. DOI PubMed

16. Albiges L, Tannir NM, Burotto M, et al. Nivolumab plus ipilimumab versus sunitinib for first-line treatment of advanced renal cell carcinoma: extended 4-year follow-up of the phase III CheckMate 214 trial. ESMO Open 2020;5:e001079. DOI PubMed PMC

17. Powles T, Plimack ER, Soulières D, et al. Pembrolizumab plus axitinib versus sunitinib monotherapy as first-line treatment of advanced renal cell carcinoma (KEYNOTE-426): extended follow-up from a randomised, open-label, phase 3 trial. Lancet Oncol 2020;21:1563-73. DOI PubMed 